\title{
Cyclophosphamide Attenuates Fibrosis in Lupus Nephritis by Regulating Mesangial Cell Cycle Progression
}

\author{
Yuehong Ma, ${ }^{1,2}$ Ling Fang, ${ }^{3}$ Rui Zhang, ${ }^{1}$ Peng Zhao, ${ }^{4}$ Yafeng Li $\mathbb{D},{ }^{1,2}$ and Rongshan Li $\mathbb{D}^{1}$ \\ ${ }^{1}$ Shanxi Key Laboratory of Kidney Disease, Department of Nephrology, Shanxi Provincial People's Hospital, Taiyuan, China \\ ${ }^{2}$ Shanxi Precision Medicine Center, Shanxi Provincial People's Hospital, Taiyuan, China \\ ${ }^{3}$ Shanxi Institute of Scientific and Technical Information, Taiyuan, China \\ ${ }^{4}$ Department of Dermatology, Shanxi Provincial People's Hospital, Taiyuan, China
}

Correspondence should be addressed to Yafeng Li; dr.yafengli@gmail.com and Rongshan Li; rongshanli163@163.com

Received 18 August 2021; Revised 13 October 2021; Accepted 21 October 2021; Published 15 November 2021

Academic Editor: Ting Su

Copyright (C) 2021 Yuehong Ma et al. This is an open access article distributed under the Creative Commons Attribution License, which permits unrestricted use, distribution, and reproduction in any medium, provided the original work is properly cited.

\begin{abstract}
Objectives. Most patients with systemic lupus erythematosus (SLE) develop lupus nephritis (LN) with severe kidney manifestations. Renal fibrosis can be primarily attributed to overproliferation of mesangial cells (MCs), which are subject to drug treatment. Nevertheless, the detailed mechanisms remain elusive. We sought to identify the effect of cyclophosphamide (CTX), a drug commonly used for LN treatment, on MC proliferation and explore its underlying mechanisms. Material/Methods. Cell proliferation and fibrosis in mouse kidney tissues were determined by histopathology staining techniques. Flow cytometry was used for cell cycle analysis. Cell cycle regulators were examined in vitro following treatment of immortalized human MCs with platelet-derived growth factor subunit B (PDGF-B). Quantitative real-time PCR and western blot analyses were used to measure the mRNA and protein levels of candidate cell cycle regulators, respectively. Results. CTX inhibited cell overproliferation induced by platelet-derived growth factor subunit B in vitro and in vivo. CTX (40 mg/l) was sufficient to induce G0/G1 phase cell cycle arrest. CTX treatment downregulated many critical cell cycle regulators including cyclins and cyclin-dependent kinases but upregulated cyclin-dependent kinase inhibitors. Additionally, CTX-treated samples showed significantly reduced fibrosis, as indicated by lower expression of interleukin- $1 \beta$ and $\alpha$-smooth muscle actin. Conclusion. CTX inhibits proliferation of MCs by modulating cell cycle regulator and therefore arresting them at G1 phase. CTX treatment significantly alleviates the severity of renal fibrosis. These findings provide novel insights into the mechanisms by which CTX affects LN.
\end{abstract}

\section{Introduction}

Systemic lupus erythematosus (SLE) is an autoimmune disease characterized by widespread inflammation in affected organs attacked by the immune system. Up to 70\% SLE patients develop renal involvement, which are clinically diagnosed as lupus nephritis (LN). LN remains a leading cause of disability and death worldwide. Chronic nephritis, nephrotic syndrome, and even acute nephritis with alternating active and stable phases are commonly seen in LN patients $[1,2]$. Additionally, 1 out 10 SLE patients ends up developing end-stage renal diseases [3,4], and LN-related renal failure is the major cause of death among patients with SLE. While the clinical characteristics of LN are complex, glomerular injury represents a predominant symptom. Glomerular injuries are histologically classified based on glomerular immune complex deposition and mesangial cell (MC) overproliferation.

The three most abundant cell types in glomeruli are MCs, podocytes, and glomeruli epithelial cells, with MCs comprising approximately $1 / 3$ of the total cell population. MC functions are vital in tissue homeostasis by providing structural support to the glomeruli and producing and maintaining the mesangial matrix. In addition, MCs can regulate the filtration surface area as a result of their contractility and can phagocytose apoptotic 
cells or immune components [5]. Finally, MCs are also involved in immune responses in the glomerulus $[6,7]$.

MC proliferation is triggered by renal cell injury or inflammation. Interfering with the cell cycle at any stage can lead to cell cycle arrest, ultimately rendering apoptosis [8]. Several studies have demonstrated that tacrolimus, cyclosporine A, and methylprednisone (MP) can inhibit the proliferation of MCs and are promising therapeutic agents in treating glomerular diseases [9-13]. Previous studies have shown that tacrolimus inhibits cell cycle progression by decreasing the percentage of cells in $\mathrm{S}$ phase and increasing the percentage of cells in G0/G1 phase [14]. Similarly, cyclosporine A also prevents human MCs from entering S phase in a dose-dependent manner. MCs exposed to low micromolar levels of cyclosporine A showed significantly increased apoptotic cells. In addition, treatment with MP inhibited the proliferation of human MCs (HMCs) in a time- and concentration-dependent manner. Similar to that with cyclosporine A, treatment with MP (1-10 $\mathrm{mg} / \mathrm{l})$ for $48 \mathrm{~h}$ also promoted HMC apoptosis [14].

Although cyclophosphamide (CTX) pulse is widely used to treat LN in clinical practice, how CTX affects cell cycle and renal fibrosis remains to be determined. $3 \mathrm{H}$-thymidine incorporation assay showed similar patterns between control and CTX-treated groups $\left(5 \times 10^{-5} \mathrm{~mol} / \mathrm{L}\right)$, suggesting that CTX did not alter the mesangial cell cycle at low concentrations [15]. However, whether high dose of CTX influences the cell cycle remains unclear. Understanding the mechanisms by which CTX affects the cell cycle and fibrosis will be useful for disease monitoring and treating $\mathrm{LN}$. We therefore initiated the present study to investigate how CTX influenced the cell cycle progression, apoptosis, and fibrosis of HMCs.

\section{Materials and Methods}

2.1. Cell Proliferation Assay. Cell Counting Kit-8 (CCK-8, Boster Biotechnology Co., Wuhan, China) was used to measure cell proliferation according to manufacturer's protocol. Briefly, HMCs were cultured in 96-well plates $\left(1 \times 10^{5}\right.$ cells/well), followed by treatment with $20 \mathrm{ng} / \mathrm{ml}$ platelet-derived growth factor subunit B (PDGF-B) for $24 \mathrm{~h}$ with or without CTX (40 or $80 \mathrm{mg} / \mathrm{l}$ ). The cells were then incubated in $10 \mu \mathrm{l}$ CCK- 8 solution at $37^{\circ} \mathrm{C}$ for $2 \mathrm{~h}$ in the dark. The optical density (OD) was measured at $450 \mathrm{~nm}$ using a Biotek microplate reader (Agilent Technologies, Santa Clara, CA, USA). The cell viability was calculated using the following formula: cell survival $(\%)=($ mean OD of treated cells/mean OD of control cells) $\times 100$.

2.2. Animals. Six young (6-7-week-old) female C57BL/6J mice weighing $20 \pm 1.5 \mathrm{~g}$ were purchased from the Laboratory Animal Center, Shanxi Provincial People's Hospital (Taiyuan, Shanxi). Female MRL/lpr LN mice were purchased from the Model Animal Institute of Nanjing University (induced from Jackson Laboratories, USA). The mice were housed under controlled environmental conditions (temperature $24 \pm 2{ }^{\circ} \mathrm{C}$, $12 \mathrm{~h}$ light-dark cycle, and humidity 40\%-70\%), given free access to water, and fed a standard laboratory diet. The study was conducted in accordance with protocols approved by the Laboratory Animal Center, Shanxi Provincial People's Hospi- tal. Twelve female MRL/lpr LN mice were randomly divided into 2 groups: LN group and LN+CTX group. Mice in the LN+CTX group were exposed to CTX by oral perfusion (20 mg/kg.d, 12 weeks), whereas the LN group received saline for 12 weeks. The animals were sacrificed $12 \mathrm{~h}$ after the last oral injection. Serum was collected by centrifuging at $5000 \mathrm{rpm}$ for $15 \mathrm{~min}$ at $4^{\circ} \mathrm{C}$ and stored at $-20^{\circ} \mathrm{C}$ for determining BUN and Scr levels. Renal tissues were obtained and kept in $10 \%$ neutral-buffered formalin and embedded in paraffin for histopathological analysis. Additional renal samples were immediately frozen in liquid nitrogen and stored at $-80^{\circ} \mathrm{C}$ until analysis.

2.3. Flow Cytometry. Cell cycle arrest after cyclophosphamide (CTX) treatment was examined by flow cytometry. The human mesangial cells (HMCs) were harvested from T25 culture flasks (Nest Biotechnology, Wuxi, China) $24 \mathrm{~h}$ after treatment and fixed with $70 \%$ ethanol at $4^{\circ} \mathrm{C}$ for $24 \mathrm{~h}$. After washing once with ice-cold phosphate-buffered saline, the cells were treated with ribonuclease $(100 \mathrm{mg} / \mathrm{ml})$ at $37^{\circ} \mathrm{C}$ for $30 \mathrm{~min}$, followed by staining with propidium iodide $(50 \mathrm{mg} / \mathrm{ml})$ at $4^{\circ} \mathrm{C}$ for $30 \mathrm{~min}$ in the dark. The cells were analyzed by using an FC500 flow cytometer (Beckman Coulter, Beckman, Palo Alto, CA, USA) to determine the proportions of cells within the G1, S, and G2/M phases.

2.4. Quantitative Real-Time Polymerase Chain Reaction ( $q R T$ PCR) Analysis. RNA was extracted from HMCs treated with $20 \mathrm{ng} / \mathrm{ml}$ PDGF-B for $24 \mathrm{~h}$. The RNA concentration was measured using a Biotek microplate reader (Agilent Technologies, Santa Clara, CA, USA). $500 \mathrm{ng}$ RNA was reversetranscribed into cDNA using PrimeScript ${ }^{\mathrm{TM}}$ RT Master Mix (Takara, Shiga, Japan) according to manufacturer's protocol. qRT-PCR was performed with TB Green ${ }^{\circledR}$ Premix Ex Taq ${ }^{\mathrm{TM}}$ II (Tli RNaseH Plus) (Takara). Primer sequences used for qRT-PCR are as follows: CDK2 F-ATCTT TGCTG AGATG GTGAC TCG and CDK2 R-ACTTG GGGAA ACTTG GCTTG T, CDK4 F-TTGCG GCCTG TGTCT ATGGT and CDK4 R-CAAGG GAGAC CCTCA CGCC, cyclin E FCCGGT ATATG GCGAC ACAAG and cyclin E R-CACAG AGATC CAACA GCTTC AT, cyclin D1 F-GATCA AGTGT GACCC GGACT and cyclin D1 R-CTTGG GGTCC ATGTT CTGCT, p21 F-ATGTG CACGG AAGGA CTTTG and p21 R-CGTTT GGAGT GGTAG AAATC TGG, and $\beta$-actin FACCTT CTACA ATGAG CTGCG and $\beta$-actin R-CCTGG ATAGC AACGT ACATG G. Relative gene expression was normalized to that of $\beta$-actin.

2.5. Western Blot Analysis. HMCs and kidney tissues were homogenized in lysis buffer (Boster Biotechnology Co., Wuhan, China). Proteins were separated by $10 \%$ SDS-PAGE and transferred onto a PVDF membrane (EMD Millipore, Billerica, MA, USA). Primary antibodies against cyclin D1, cyclin E, CDK2, CDK4, p21 (Santa Cruz Biotechnology, Dallas, TX, USA), IL-1 $\beta$ (ab234437; Abcam, Cambridge, UK), and $\alpha$ smooth A (ab5694; Abcam) and corresponding secondary antibodies (Boster Biotechnology Co., Wuhan, China) were used. Membranes were developed and visualized using the Quantity One analysis system (Bio-Rad, Hercules, CA, USA). 
2.6. Kidney Tissue Preparation for Pathology. Kidney tissues were collected from anesthetized mice after perfusion. To prepare sliced sections for histology, kidney tissue was fixed with $4 \%$ paraformaldehyde, embedded in paraffin, cut to $4 \mu \mathrm{m}$ thickness, and stained with hematoxylin and eosin (HE) or periodic acid-Schiff (PAS) for morphological evaluation, PAS for mesangial expansion, and Masson for fibrosis. This study was approved by the local Ethics Committee of Shanxi Provincial People's Hospital, China (no.: 201987).

2.7. Data Analyses. Experimental data with three biological repeats were analyzed statistically by paired $t$-tests using the SPSS 23.0 software (SPSS, Inc., Chicago, IL, USA) and are presented as the means \pm standard error of the mean.

\section{Results}

3.1. CTX Reduces Proteinuria and Improves Renal Function in MRL/lpr Mice. Treatment of MRL/lpr mice with 40 or $80 \mathrm{mg} / \mathrm{l} \mathrm{CTX}$ ameliorated the symptoms of LN. Specifically, proteinuria, renal function abnormities, and kidney pathological lesions were reduced. The reduction in proteinuria was similar between the two dosage groups (Figure 1(a)).

3.2. CTX Inhibits Cellular Proliferation in Glomeruli In Vivo. To examine the cellular effect of CTX treatment on mice with $\mathrm{LN}$, we have performed $\mathrm{HE}$ and PAS staining. Compared to the control mice, CTX-treated mice showed increased MC proliferation and accelerated progression of kidney fibrosis at week 12 . We also examined both groups at week 17 , and the difference was found to be more significant. Remarkably, mice treated with CTX for 12 weeks showed delayed disease progression compared to untreated mice. Pathological evaluation of MRL/lpr mice at week 17 revealed glomeruli with thickened pink capillary loops, which are typical of the so-called "wire loops" of LN. In mice treated with CTX, the surrounding renal tubules were unremarkable, and the opening of capillary loops was improved (Figure 1(b)).

3.3. MCs Treated with CTX Contribute to Decreased Inflammation and Fibrosis in LN. Masson staining showed that CTX-treated mice delayed the progress of renal fibrosis, compared to the MRL/lpr LN mice (Figure 2(a)). And meanwhile, we investigated how CTX affects cellular inflammation and fibrosis. Interleukin- (IL-) $1 \beta$ is a key cytokine mediating inflammatory responses while $\alpha$-smooth muscle actin $(\alpha$-SMA) is a marker for tissue fibrogenesis. IL- $1 \beta$ was expressed at relatively high levels in LN samples, indicating their inflammatory origins. However, as shown by the qRT-PCR and western blot results, IL- $1 \beta$ levels were significantly reduced following treatment with CTX, suggesting an ameliorated inflammatory response. $\alpha$-SMA showed the same tendency following CTX treatment (Figure 2(b)). These results suggested that CTX could potentially mitigate both inflammation and fibrosis in LN patients.

3.4. CTX Reduces HMC Proliferation and Arrests the Cell Cycle in G0/G1 Phase. We next assessed how CTX influences HMC proliferation in vitro. Cellular proliferation in CTX and PDGF-B-treated group was significantly reduced compared to that in cells treated by PDGF-B alone. We also found that the inhibitory effect of CTX depended not only on its dosage but also on its exposure time, although the difference was negligible at the high concentration. We found that neither 40 nor $80 \mathrm{mg} / \mathrm{l}$ CTX affected HMC proliferation following a 24-hour treatment. However, the inhibition rate of cell proliferation has the similar tendency with $24 \mathrm{~h}$ after treatment with 40 and $80 \mathrm{mg} / \mathrm{l} \mathrm{CTX}$ for $48 \mathrm{~h}$, but has significance in statistics (Figure 3(a)).

One plausible explanation of the reduced HMC proliferation upon CTX exposure was the shifted cell cycle. Therefore, we performed flow cytometry analysis to test this hypothesis. Consistent with our results of cellular proliferation, PDGF-B treatment increased the proportion of cells in $S$ phase while decreasing the percentage of G1 cells. These results indicated that PDGF-B could shift cell cycle towards $S$ phase (Figure 3(b)). In contrast, cells exposed to both PDGF-B and CTX were significantly enriched for the G1 phase population $(p<0.05)$, while deprived of the $S$ phase population $(p<0.0001)$. These results suggested that CTX could override PDGF in halting the cells in G1 phase and blocking cell cycle.

3.5. Effects of CTX on Cyclins and Cyclin-Dependent Kinases. Cyclins and cyclin-dependent kinases (CDKs) are essential drivers of cell cycle events. To determine the mechanisms by which CTX affects HMC proliferation, we examined the expression of genes that are known to regulate G1 phase, such as cyclin D1, cyclin E, CDK2, and CDK4 (Figure 4). Both transcription and protein levels of the candidate factors were upregulated when cells were treated with $20 \mathrm{ng} / \mathrm{l}$ PDGF-B. Significantly, we found that treatment with CTX at $40 \mathrm{mg} / \mathrm{l}$ was sufficient to abolish the upregulation induced by PDGF-B. We also examined the effect of high dosage of CTX and found that $80 \mathrm{mg} / \mathrm{l} \mathrm{CTX}$ inhibited the expression of all the proteins except for CDK4, which showed higher levels than that in PDGF-B-treated cells (Figure 4(a)).

3.6. CTX Reverses the PDGF-B-Induced Decrease in $p 21$ Level. To gain further insights into how CTX regulates cell cycle components, we focused on p21, a cyclin-dependent kinase inhibitor, in quiescent and proliferating HMCs. In quiescent HMCs, both the mRNA and protein levels of p21 were detectable. However, the expression of p21 was decreased in cells incubated with PDGF-B. Significantly, this effect was reversed by $40 \mathrm{mg} / \mathrm{l}$ CTX treatment. Interestingly, treatment with $80 \mathrm{mg} / \mathrm{l}$ CTX only decreased p21 mRNA expression but not protein expression (Figure 4(b)). Altogether, these results suggested that CTX can reverse the downregulation of cyclin-dependent kinase inhibitor induced by PDGF-B exposure.

\section{Discussion}

Many SLE patients develop LN that is associated with poor prognosis and increased morbidity and mortality [16]. One shared phenotype of many glomerular diseases, including LN, is MC overproliferation [12]. MCs are involved in many 

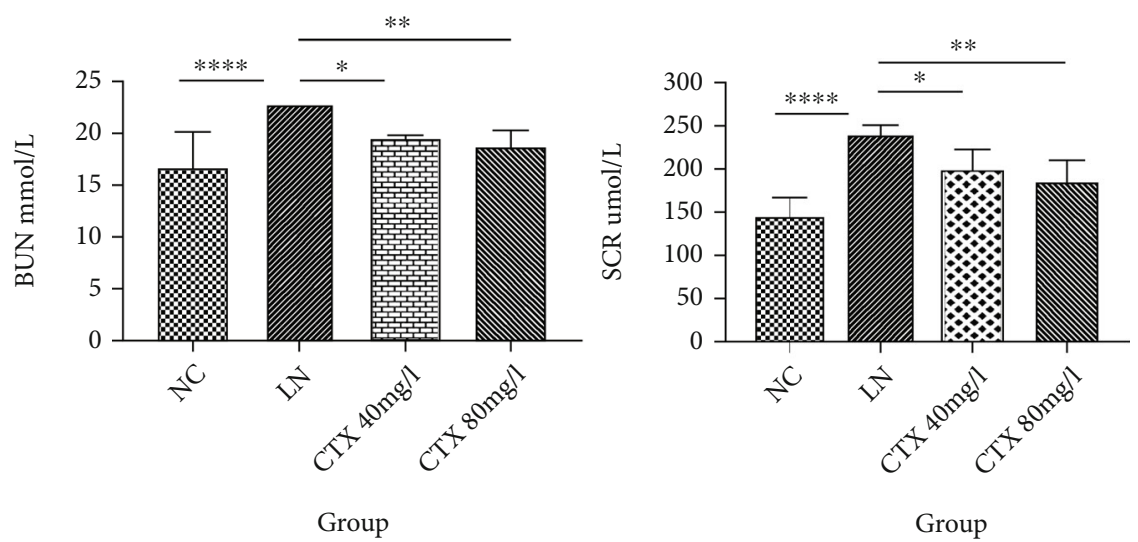

$$
\begin{aligned}
& \text { NC } \\
& \text { LN } \\
& \text { CTX } 40 \mathrm{mg} / 1 \\
& \text { CTX } 80 \mathrm{mg} / 1
\end{aligned}
$$$$
\begin{aligned}
& \infty \mathrm{NC} \\
& m \mathrm{LN} \\
& \square \mathrm{CTX} 40 \mathrm{mg} / \mathrm{l} \\
& \text { CTX } 80 \mathrm{mg} / \mathrm{l}
\end{aligned}
$$

(a)
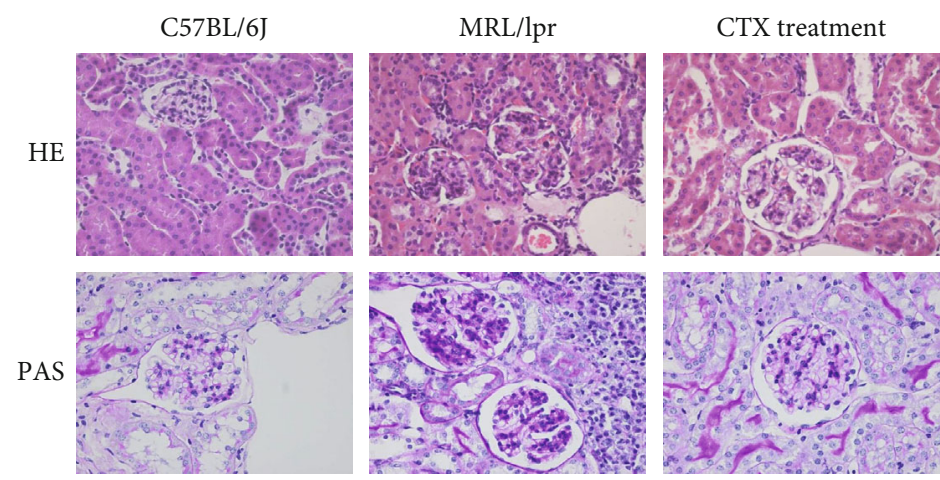

(b)

FIgURE 1: (a) Renal function in lupus nephritis after CTX treatment. CTX reduced the levels of blood urea nitrogen (BUN) and serum creatinine (SCR) of lupus nephritis (LN) mice. Data are presented as the means \pm SEM $(n=6) .{ }^{*} p<0.05,{ }^{* *} p<0.001$, and ${ }^{* * * *} p<$ 0.00001, lupus nephritis vs. control. (b) CTX $40 \mathrm{mg} / \mathrm{l}$ inhibits cell proliferation in glomeruli and necrosis of capillary loops. Glomerular pathology was detected by HE staining and PAS staining at week 17 ( $n=6 /$ group, 400x original magnification).

biological processes in the renal glomerulus, such as secreting cell matrix, producing cytokines, and supporting glomerular capillary plexus, phagocytosis, and clearance of macromolecular substances, as well as contraction of smooth muscle cells. Once activated by inflammatory stimuli, MCs can also interact with migrating and infiltrating inflammatory cells, which in turn amplifies local inflammation responses, fibrosis, and the development of glomerulosclerosis $[17,18]$.

Similar to tacrolimus and cyclosporine A, MP is known to interfere with cell cycles by inducing G1-phase arrest and preventing cells from entering mitosis [14]. Consistent with this result, another study showed that glucocorticoids could decrease S/G2/M-phase populations in HEK293 cells by suppressing NF- $\kappa \mathrm{B}$ activities [19]. Mounting evidence has shown the antiproliferative effects of glucocorticoids across numerous cell types [20-24], and MP is likely to be effective on CDK inhibitors such as p21/Cip1 and p57/Kip2 [25, 26]. Alternatively, MP may also suppress the expression of c-myc or cyclins, which can stimulate cell cycle progression [20].
To dive into the prime feature of LN, we first analyzed the effects of CTX treatment on cell proliferation in vitro. Cell cycle progression is tightly regulated and coordinated by growth factors, oncogenic stimuli, and regulatory components such as cyclin/CDK complexes [27, 28]. Our data showed that the cell cycle was shifted by treating MCs with $40 \mathrm{mg} / \mathrm{l}$ or $80 \mathrm{mg} / \mathrm{l} \mathrm{CTX}$. As our results indicated that CTX mainly affected G0/G1 phase, we focused on regulators of these stages. Cyclin D and cyclin E, together with CDK2 and CDK4, facilitate the transition from G1 to G2 phases, whereas p21 has a strong inhibitory effect. Our results showed that PDGF-B could upregulate cyclin D, cyclin E, CDK2, and CDK4, while the expression of p21 was markedly reduced. Interestingly, in proliferating MCs, CTX downregulated the expression of cyclin D, cyclin E, CDK2, and CDK4 and upregulated p21 expression. These results suggested an antiproliferative effect of CTX in MCs.

An in vitro model of LN suggested that MCs could contribute to renal inflammation by secreting proinflammatory 

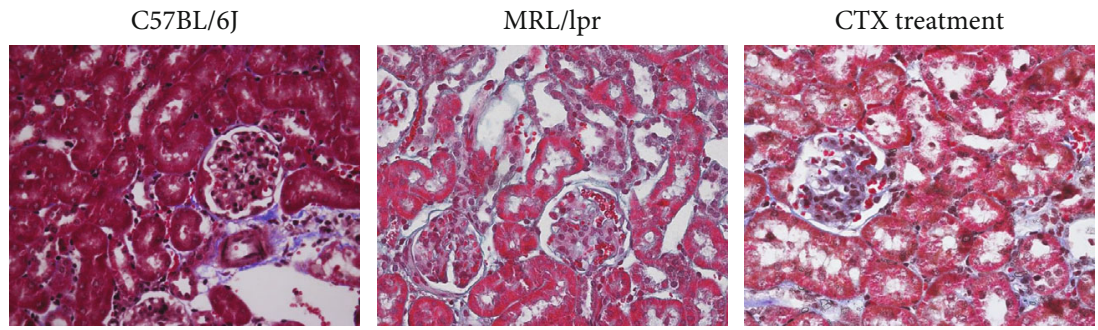

(a)
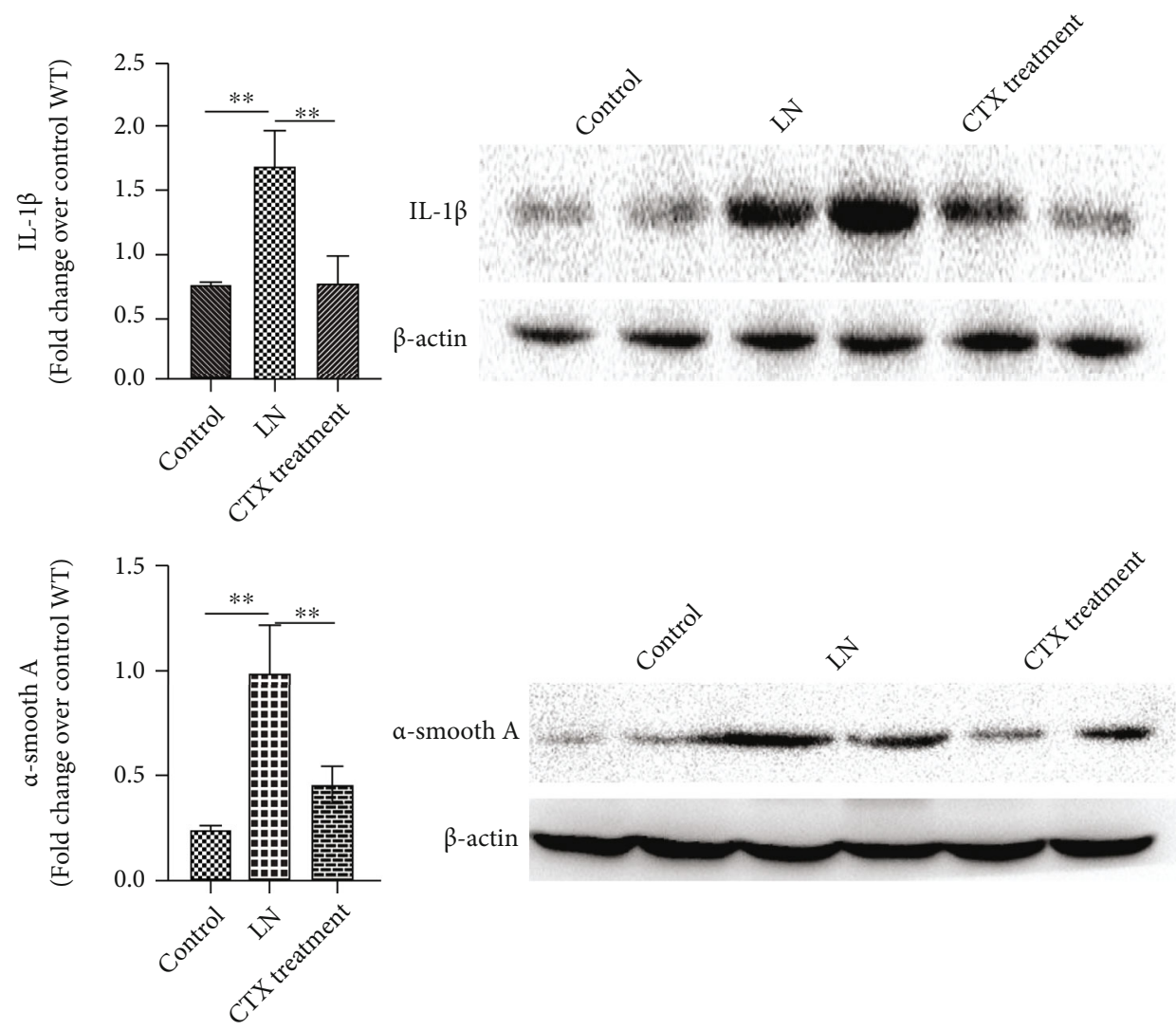

(b)

FIgURE 2: (a) CTX $40 \mathrm{mg} / \mathrm{l}$ delays the progress of kidney fibrosis. Glomerular pathology was detected by Masson staining at week 17 ( $n=6$ /group, 400x original magnification). (b) Interleukin- (IL-) $1 \beta$ and $\alpha$-SMA in LN following CTX (40 mg/l) treatment. Multiplex analysis was used to determine the protein levels of IL-1 $\beta$ and $\alpha$-SMA. The blots were representatives of independent biological triplicates and were analyzed by Friedman's test with Dunn's post hoc test, ${ }^{* *} p<0.01$.

cytokines to local niches [7]. Studies based on human and animal models also showed that renal tubular epithelialmyofibroblast phenotypic transformation is critical in the pathogenesis of renal diseases associated with renal interstitial fibrosis, where $\alpha$-SMA is an important marker of this transformation [29]. Additionally, fibrosis and key inflammatory factors, such as IL-1 $\beta$, appear to be closely related [30].

Our study indicated that CTX could benefit renal fibrosis patients by targeting key cell cycle regulators. We found that expression of the fibrotic protein $\alpha$-SMA and inflammatory factor IL- $1 \beta$ was markedly reduced in the LN mouse model after treatment with CTX, which delayed the progression of renal fibrosis and inflammation. Although we did not observe the same impact of CTX on HMCs, this discrepancy can be explained by the different concentrations of CTX administered in vitro and in vivo. While CTX $\left(5 \times 10^{-5} \mathrm{~mol} / \mathrm{L}\right) \mathrm{did}$ not alter the mesangial cell cycle [15], these data reveal a strong correlation between cell cycle, inflammation, and fibrosis. As an important hub of LN-related phenotypes including cell proliferation and immune cell activation, MC remains to be a promising therapeutic target. Our work elucidated detailed mechanisms by which CTX attenuates MC overproliferation and renal fibrosis and therefore laid a foundation for CTX therapy. 


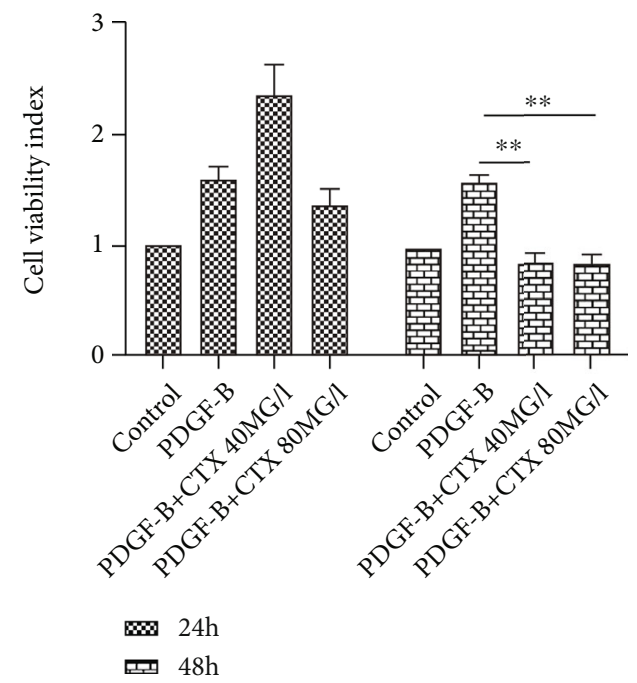

(a)
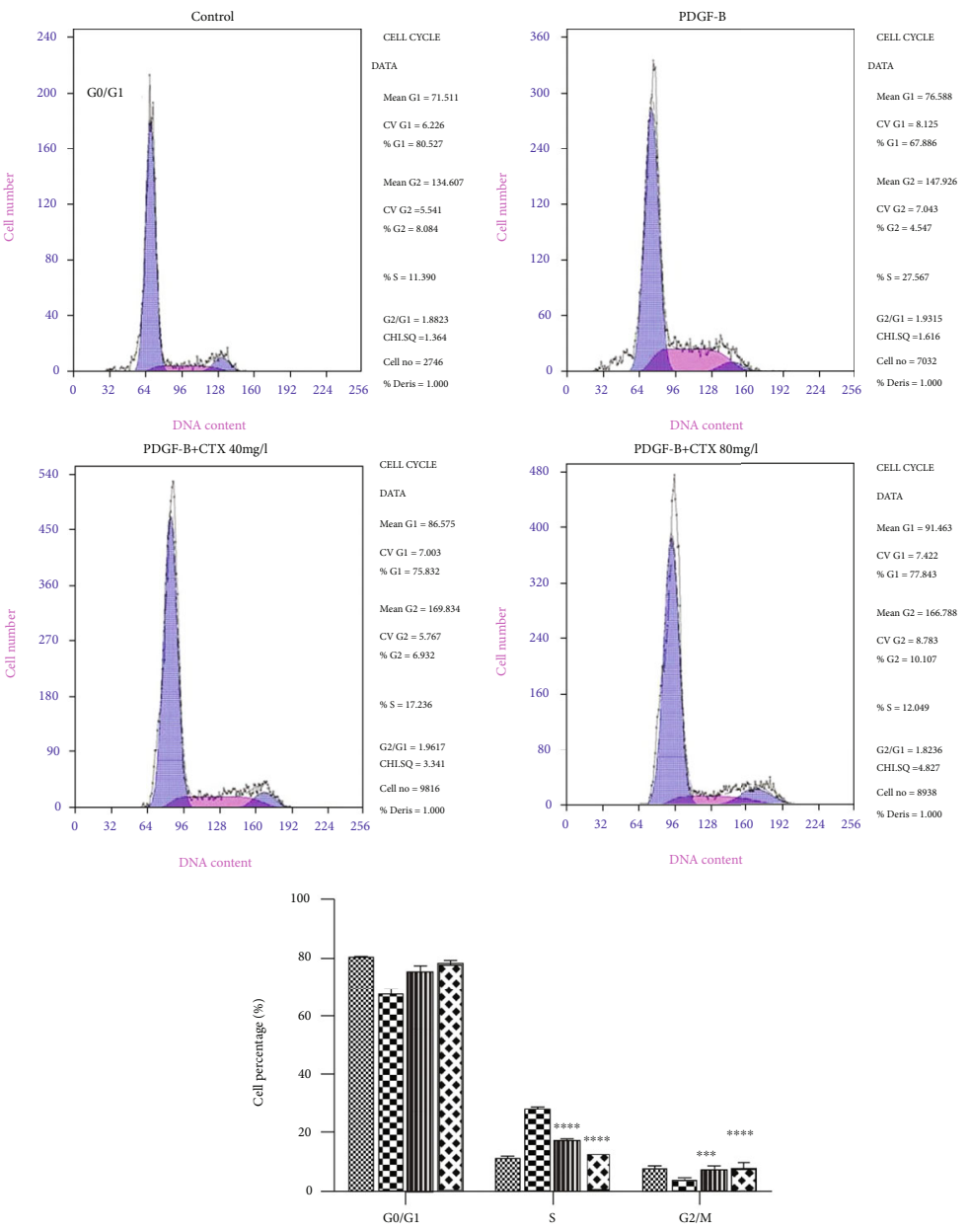

G0/G

$\mathrm{G} 2 / \mathrm{M}$

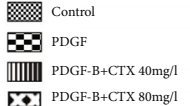

(b)

FIGURE 3: CTX reduces HMC proliferation by arresting the cell cycle in G1 phase. (a) CTX inhibited cell proliferation induced by PDGF-B at $48 \mathrm{~h}$ but not $24 \mathrm{~h}$ ( $n=6 /$ group). (b) Flow cytometry analysis showing cells in different phases of cell cycle. CTX arrested cells in G1 phase in a concentration-dependent manner; therefore, the percentage of cells in S phase was significantly lower $(n=6 /$ group). Data are presented as the means $\pm \mathrm{SD},{ }^{*} p<0.05,{ }^{* * *} p<0.001$, and ${ }^{* * * *} p<0.0001$. 


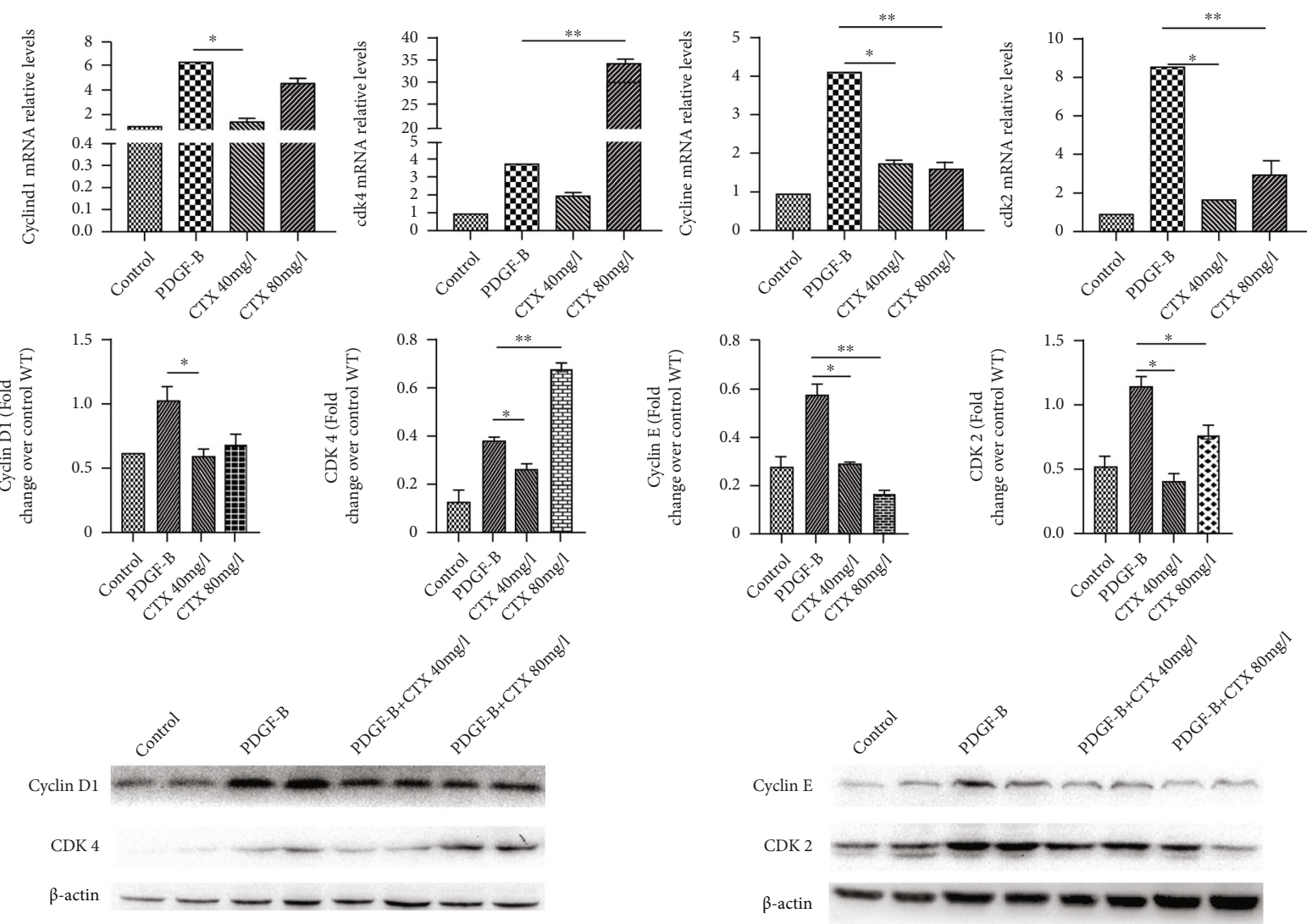

(a)
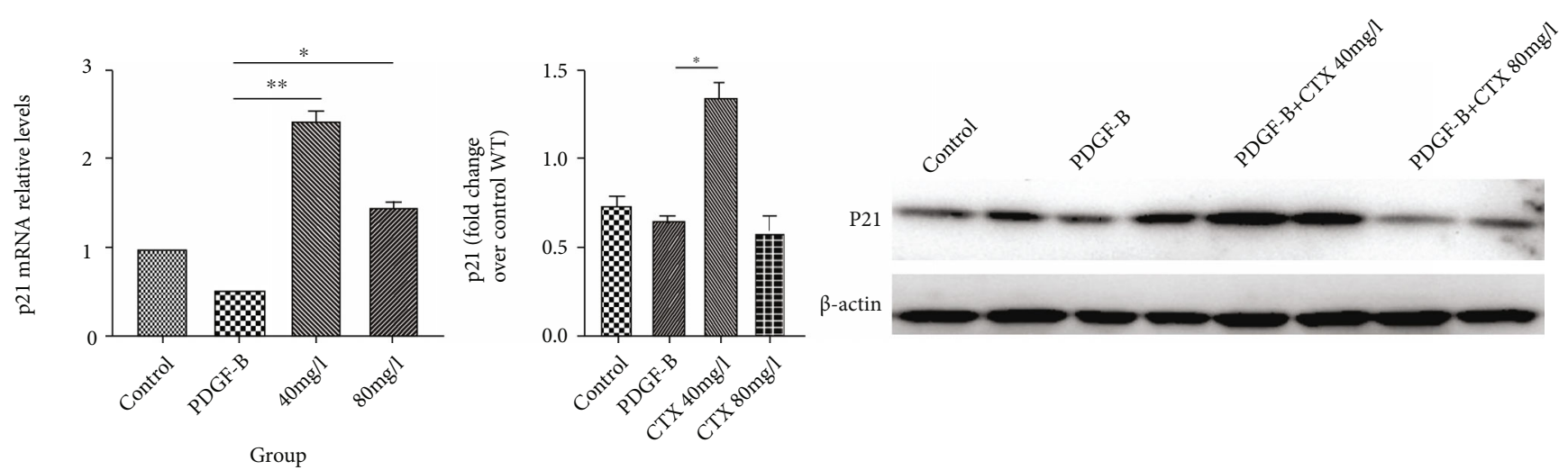

(b)

Figure 4: (a) Effect of CTX on candidate genes. HMCs were treated with $20 \mathrm{ng} / \mathrm{ml}$ PDGF-B for $48 \mathrm{~h}$ with or without CTX (40 or $80 \mathrm{mg} / \mathrm{l})$. The expression levels of candidate genes were detected by western blotting and qRT-PCR. Blots were representatives of independent biological triplicates. ${ }^{*} p<0.05$ and ${ }^{* *} p<0.01$. Data are presented as the means \pm SD $(n=3)$. (b) Effect of CTX on cell cycle proteins. CTX (40 and $80 \mathrm{mg} / \mathrm{l}$ ) upregulated p21 mRNA and protein in HMCs as demonstrated by qRT-PCR and western blotting assays, respectively. The western blots were representatives of independent biological triplicates. ${ }^{*} p<0.05$ and ${ }^{* *} p<0.01$. Data are presented as the means $\pm \mathrm{SD}(n=3)$. 


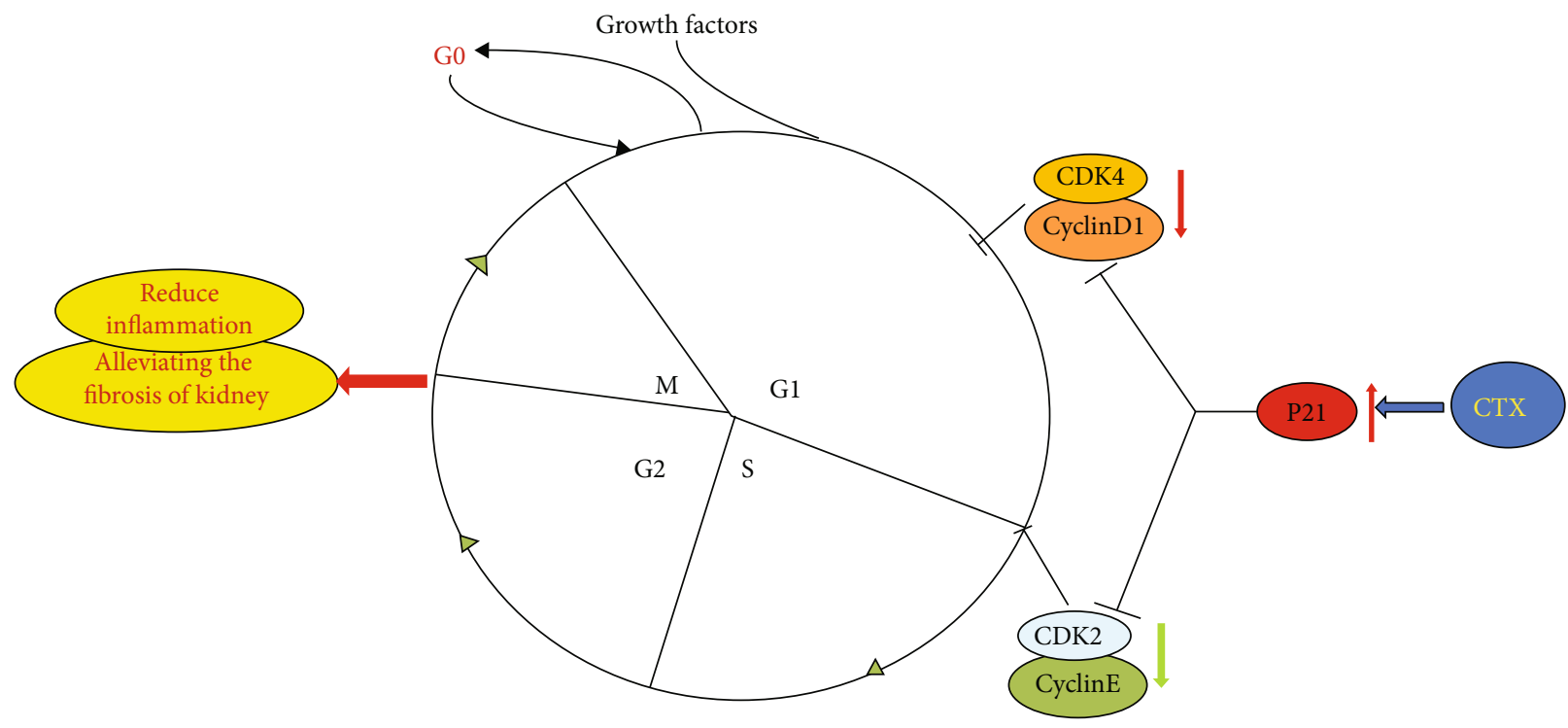

Figure 5: Proposed mechanisms of effects of CTX on mesangial cell proliferation.

\section{Conclusion}

In conclusion, a high dose of CTX inhibited the proliferation of HMCs and induced HMC apoptosis. Moreover, CTX dramatically reduced the expression of IL- $1 \beta$ and $\alpha$-SMA, which are involved in the inflammatory response and fibrosis within the glomerulus. Therefore, we proposed that CTX might regulate cell proliferation by controlling the production of intracellular inflammatory fibrosis mediators and the cell cycle regulators. Overall, these data laid the foundation of CTX shock therapy for LN (Figure 5).

\section{Data Availability}

The data used to support the findings of this study are available from the first author upon request.

\section{Conflicts of Interest}

We do not have any competing interest to declare.

\section{Authors' Contributions}

Yuehong Ma and Ling Fang contributed equally to this work.

\section{Acknowledgments}

This work was financially supported by the National Natural Science Foundation of China (grant number: 81570626), Foundation of Health Commission of Shanxi Province of China (grant number: 2020033), and Social Development Project of Science and Technology Department of Shanxi Province of China (grant number: 201803D31158).

\section{References}

[1] J. H. Berden, "Lupus nephritis," Kidney International, vol. 52, no. 2, pp. 538-558, 1997.

[2] G. B. Appel and A. Valeri, "The course and treatment of lupus nephritis," Annual Review of Medicine, vol. 45, no. 1, pp. 525537, 1994.

[3] S. Almaani, A. Meara, and B. H. Rovin, "Update on lupus nephritis," Clinical Journal of the American Society of Nephrology, vol. 12, no. 5, pp. 825-835, 2017.

[4] M. G. Tektonidou, A. Dasgupta, and M. M. Ward, "Risk of end-stage renal disease in patients with lupus nephritis, 1971-2015: a systematic review and Bayesian meta-analysis," Arthritis \& Rhematology, vol. 68, no. 6, pp. 1432-1441, 2016.

[5] H. E. Abboud, "Mesangial cell biology," Experimental Cell Research, vol. 318, no. 9, pp. 979-985, 2012.

[6] D. Schlondorff, "Roles of the mesangium in glomerular function," Kidney International, vol. 49, no. 6, pp. 1583-1585, 1996.

[7] R. D. Wright, P. Dimou, S. J. Northey, and M. W. Beresford, "Mesangial cells are key contributors to the fibrotic damage seen in the lupus nephritis glomerulus," Journal of Inflammation, vol. 16, no. 1, pp. 1-4, 2019.

[8] O. Pastukhov, S. Schwalm, I. Romer, U. Zangemeister-Wittke, J. Pfeilschifter, and A. Huwiler, "Ceramide kinase contributes to proliferation but not to prostaglandin E2 formation in renal mesangial cells and fibroblasts," Cellular Physiology and Biochemistry, vol. 34, no. 1, pp. 119-133, 2014.

[9] E. S. Akool, A. Doller, A. Babelova et al., "Molecular mechanisms of TGF $\beta$ receptor-triggered signaling cascades rapidly induced by the calcineurin inhibitors cyclosporin A and FK506," Journal of Immunology, vol. 181, no. 4, pp. 2831$2845,2008$.

[10] M. S. Anil Kumar, M. Irfan Saeed, K. Ranganna et al., "Comparison of four different immunosuppression protocols without long-term steroid therapy in kidney recipients monitored by surveillance biopsy: Five- year outcomes," Transplant Immunology, vol. 20, no. 1-2, pp. 32-42, 2008. 
[11] J. Boletis, A. Balitsari, V. Filiopoulos et al., "Delayed renal graft function: the influence of immunosuppression," Transplantation Proceedings, vol. 37, no. 5, pp. 2054-2059, 2005.

[12] Y. Kurogi, "Mesangial cell proliferation inhibitors for the treatment of proliferative glomerular disease," Medicinal Research Reviews, vol. 23, no. 1, pp. 15-31, 2003.

[13] H. Ren, N. Guo, and D. Lu, "Successful engraftment of HLAidentical sibling cord blood transplantation in an adult with chronic myelogenous leukemia," Zhonghua Xue Ye Xue Za Zhi, vol. 22, no. 12, pp. 621-624, 2001.

[14] X. Zhou, B. Workeneh, Z. Hu, and R. Li, "Effect of immunosuppression on the human mesangial cell cycle," Molecular Medicine Reports, vol. 11, no. 2, pp. 910-916, 2015.

[15] D. R. Cha, S. M. Feld, C. Nast, J. LaPage, and S. G. Adler, “Apoptosis in mesangial cells induced by ionizing radiation and cytotoxic drugs," Kidney International, vol. 50, no. 5, pp. 1565-1571, 1996.

[16] R. Mina and H. I. Brunner, "Pediatric lupus-are there differences in presentation, genetics, response to therapy, and damage accrual compared with adult lupus?," Rheumatic Diseases Clinics of North America, vol. 36, no. 1, pp. 53-80, 2010, viiviii.

[17] R. L. Pereira, R. J. Felizardo, M. A. Cenedeze et al., "Balance between the two kinin receptors in the progression of experimental focal and segmental glomerulosclerosis in mice," Disease Models \& Mechanisms, vol. 7, no. 6, pp. 701-710, 2014.

[18] W. Hu, Y. Chen, S. Wang et al., "Clinical-morphological features and outcomes of lupus podocytopathy," Clinical Journal of the American Society of Nephrology, vol. 11, no. 4, pp. 585592, 2016.

[19] L. G. Bladh, J. Lidén, A. Pazirandeh et al., "Identification of target genes involved in the antiproliferative effect of glucocorticoids reveals a role for nuclear factor-(kappa)B repression," Molecular Endocrinology, vol. 19, no. 3, pp. 632-643, 2005.

[20] I. Rogatsky, J. M. Trowbridge, and M. J. Garabedian, "Glucocorticoid receptor-mediated cell cycle arrest is achieved through distinct cell-specific transcriptional regulatory mechanisms," Molecular and Cellular Biology, vol. 17, no. 6, pp. 3181-3193, 1997.

[21] E. Smith, R. A. Redman, C. R. Logg, G. A. Coetzee, N. Kasahara, and B. Frenkel, "Glucocorticoids inhibit developmental stage-specific osteoblast cell cycle. Dissociation of cyclin A-cyclin-dependent kinase 2 from E2F4-p130 complexes," The Journal of Biological Chemistry, vol. 275, no. 26, pp. 19992-20001, 2000.

[22] K. Rhee, D. Reisman, W. Bresnahan, and E. A. Thompson, "Glucocorticoid regulation of G1 cyclin-dependent kinase genes in lymphoid cells," Cell Growth \& Differentiation, vol. 6, no. 6, pp. 691-698, 1995.

[23] A. Helmberg, N. Auphan, C. Caelles, and M. Karin, "Glucocorticoid-induced apoptosis of human leukemic cells is caused by the repressive function of the glucocorticoid receptor," The EMBO Journal, vol. 14, no. 3, pp. 452-460, 1995.

[24] I. Sanchez, L. Goya, A. K. Vallerga, and G. L. Firestone, "Glucocorticoids reversibly arrest rat hepatoma cell growth by inducing an early G1 block in cell cycle progression," Cell Growth \& Differentiation, vol. 4, no. 3, pp. 215-225, 1993.

[25] S. Corroyer, E. Nabeyrat, and A. Clement, "Involvement of the cell cycle inhibitor CIP1/WAF1 in lung alveolar epithelial cell growth arrest induced by glucocorticoids," Endocrinology, vol. 138, no. 9, pp. 3677-3685, 1997.
[26] H. H. Cha, E. J. Cram, E. C. Wang, A. J. Huang, H. G. Kasler, and G. L. Firestone, "Glucocorticoids stimulate p21 gene expression by targeting multiple transcriptional elements within a steroid responsive region of the p21waf1/cip1 promoter in rat hepatoma cells," The Journal of Biological Chemistry, vol. 273, no. 4, pp. 1998-2007, 1998.

[27] L. Hilakivi-Clarke, C. Wang, M. Kalil, R. Riggins, and R. G. Pestell, "Nutritional modulation of the cell cycle and breast cancer," Endocrine-Related Cancer, vol. 11, no. 4, pp. 603$622,2004$.

[28] T. Li, X. Zhao, Z. Mo et al., "Formononetin promotes cell cycle arrest via downregulation of Akt/cyclin D1/CDK4 in human prostate cancer cells," Cellular Physiology and Biochemistry, vol. 34, no. 4, pp. 1351-1358, 2014.

[29] G. S. Jung, Y. J. Hwang, J. H. Choi, and K. M. Lee, "Lin28a attenuates TGF-beta-induced renal fibrosis," BMB Reports, vol. 53, no. 11, pp. 594-599, 2020.

[30] S. C. W. Tang and W. H. Yiu, "Innate immunity in diabetic kidney disease," Nature Reviews. Nephrology, vol. 16, no. 4, pp. 206-222, 2020. 